\title{
Modeling of Film Thickness and Traction in a Variable Ratio Traction Drive Rig
}

K. J. Sharif

H. P. Evans

R. W. Snidle

School of Engineering, Cardiff University,

Cardiff, U.K.

J. P. Newall Torotrak (Development) Ltd., Leyland, U.K.
The paper describes the application of a full non-Newtonian, thermal elastohydrodynamic lubrication (EHL) model for the prediction of film thickness and viscous traction force in a special high speed rolling traction rig. The primary objective of the work was to identify a suitable lubricant rheological model that would describe the behavior of practical EHL traction drive contacts over their operating range. Experiments were carried out on a special rolling contact rig at temperatures of 60,90 , and $120^{\circ} \mathrm{C}$ and contact loads giving maximum Hertzian pressures of 1, 2, and 3 GPa. Entrainment speeds of up to $18 \mathrm{~m} / \mathrm{s}$ were investigated. Corresponding modeling work was carried out using lubricant physical properties obtained for Santotrac 50, the traction fluid used in the experiments. Viscosity data for this lubricant were available from the work of Bair and Winer, but a degree of extrapolation was required to this data to cover the range of the experiments. In view of the crucial importance of viscosity/pressure behavior in the prediction of traction attention was therefore focused upon the lower contact loads for which reliable viscosity/ pressure data are available. A best-fit exercise was then carried out to establish an appropriate rheological model to account for shear thinning of the lubricant. Different non-Newtonian relationships were investigated including those of Johnson and Tevaarwerk, Bair and Winer, and a model which combined the features of both of these. The most encouraging agreement between experiment and theory over the range of temperatures and speeds considered was obtained with the Johnson and Tevaarwerk (Eyring) model. [DOI: 10.1115/1.1609490]

\section{Introduction}

Elastohydrodynamic lubrication (EHL) is the mechanism that protects the surfaces of concentrated contacts such as those occurring in gears and rolling element bearings. In an EHL contact lubricant is drawn into the conjunction between the surfaces which are then separated by a thin film of fluid. The thickness of the film is governed by the relative geometry of the surfaces and the speed with which they roll together. A crucially important beneficial effect is that of pressure upon the viscosity of the lubricant. At the pressures occurring in real contacts (typically $1 \mathrm{GPa}$ and higher) the viscosity of most lubricants is typically increased by several orders of magnitude. This leads to the generation of films that are extremely stiff and are sufficiently thick to effectively separate the surfaces and protect them from wear and damage. When relative sliding occurs between the surfaces high shear forces are set up in the lubricant film and this is the source of the observed "friction" between gear teeth for example. In lubricated traction drives this fluid friction provides the desired tangential force that is transmitted between the traction components. The EHL traction force is exploited in variable ratio torroidal drives which are used at the heart of infinitely variable automatic transmission systems. By smoothly optimizing the output of a vehicle engine to driver demand such variator transmissions can give fuel savings of $20 \%$ or more [1]. In these applications it is desirable to achieve as high a traction force as possible at low degrees of slip in order to maximize the efficiency of the device as a whole. The work described in this paper is concerned with determining the relation between traction force and slip experimentally over a

Contributed by the Tribology Division of THE AMERICAN SOCIETY OF MECHANICAL ENGINEERS for presentation at the STLE/ASME Joint International Tribology Conference, Ponte Vedra, FL October 26-29, 2003. Manuscript received by the Tribology Division February 12, 2003 revised manuscript received June 26, 2003. Associate Editor: M. R. Lovell. wide range of conditions, and also with the development of a rheological model of the traction fluid that can be used to predict traction behavior in real variator contacts.

Investigation of traction in EHL contacts has a distinguished history with the works of Dyson [2], and Hirst and Moore [3] seen as important landmarks. Traction experiments were reported by Johnson and Tevaarwerk [4] who drew attention to the need for non-Newtonian modelling of lubricants in traction contacts. Early analyses of non-Newtonian behavior in EHL contacts were provided by Conry et al. [5] for the line contact (one-dimensional) situation and by Kim and Sadeghi [6] for point (two-dimensional) contacts. In EHL traction contacts heat is inevitably generated by shearing of the oil film due to the relative sliding of the contacting surfaces. Consequently any realistic model of traction behavior must take into account the temperature rise in the fluid film due to shearing and the effect which this has upon the fluid properties (principally viscosity).

In practically useful traction devices the degree of slip must be limited to very small values with slide/roll ratios of typically $1 \%$. A feature that can become of importance under such a condition is that of "spin" at the contact arising from the kinematic design of the traction device. Spin introduces an unwanted, additional degree of slip, which can significantly reduce the efficiency of the contact. Spin also complicates the non-Newtonian numerical modeling of EHL contacts due to the fact that the local sliding vector is oriented at a varying angle to the entrainment or rolling velocity direction. In a previous paper [7] the authors have shown the need to set up the fundamental relationship between flow and pressure gradient in the sliding and non-sliding directions and have also incorporated a full thermal analysis into the solution scheme. This approach was developed for analysis of worm gears, and is a prerequisite for an accurate appraisal of the traction behavior in torroidal variator contacts where spin is an unavoidable kinematic property. 
The Johnson and Tevaarwerk rheological model [4] is based upon the analysis of the results of EHL traction experiments carried out in a disk machine. In this experimental configuration the shear rate varies within the contact due to the variation of film thickness, and the underlying low shear rate viscosity also varies greatly due to pressure and temperature variations within the Hertzian contact area. Consequently some assumptions were made by the authors regarding the pressure, film thickness and temperature distribution in the experiments in order to determine the nonNewtonian lubricant parameters. A form of differential heating of the contacting components was adopted in these experiments in an attempt to minimize temperature variation at different sliding speeds. In spite of such measures, however, disk experiments of this type are inevitably limited to the determination of average shear stress over the contact area in the form of the experimental traction force and cannot reveal the fundamental shear stress/shear strain rate characteristic.

A different approach to characterizing the lubricant's nonNewtonian behavior has been pursued by Bair and Winer in a number of publications, and they propose a limiting shear stress model for the lubricant [8]. Their approach has been to develop special apparatus to produce high pressure conditions in the lubricant without the use of EHL contacts. In this way they impose a controlled shear rate on a fluid held at constant elevated pressures and temperatures. They have also contributed key information in the form of low shear rate viscosity measurements at elevated pressures and temperatures [9] which represent the best available data at the present time.

The traction drives under development by the industrial partner in the joint research effort reported here use crowned traction rollers that are loaded against torroidal disks with contact pressures in excess of $2 \mathrm{GPa}$. The contact areas are quite large compared to those typically used in disk machine experiments and they have a degree of inherent spin due to the kinematics of the device. To screen potential traction fluids and undertake systematic testing a special traction test rig, which simulates the full range of conditions encountered by the traction rollers, has been developed. The range of conditions exceeds those typically used in laboratory traction experiments in the past. In this paper experimental traction curves obtained on this rig are presented for one particular well-documented traction fluid. The process by which numerical thermal EHL models were then used to replicate the observed traction behavior is described, and results presented showing the best-fit models obtained using both the Johnson and Tevaarwerk [4] and Bair and Winer [8] non-Newtonian fluid models.

\section{Traction Measurement Rig}

As part of the core experimental program a large-scale traction rig was developed, specifically for the measurement of the traction behavior of traction fluids under conditions representative of the contacts in a full toroidal variator [10]. The traction rig was based on the Plint TE73 twin disk rig [11], but the design was considerably modified to improve its capabilities for this application. The changes made ensure that no elements of the contact normal load are resolved in the direction of the traction force, since the original design of the rig was found to be problematic in this respect. The speed and load range of the rig were also increased significantly compared to the TE73 to facilitate contact conditions similar to those seen in a full toroidal variator.

The general arrangement of the test head of the rig is shown schematically in Fig. 1. The upper and lower rollers of the rig have a spherical running track and are each independently driven by a $75 \mathrm{~kW}$ speed controlled electric motor about parallel rotational axes AA and CC. By accurately maintaining the motor speeds relative to one another, slip may be introduced into the

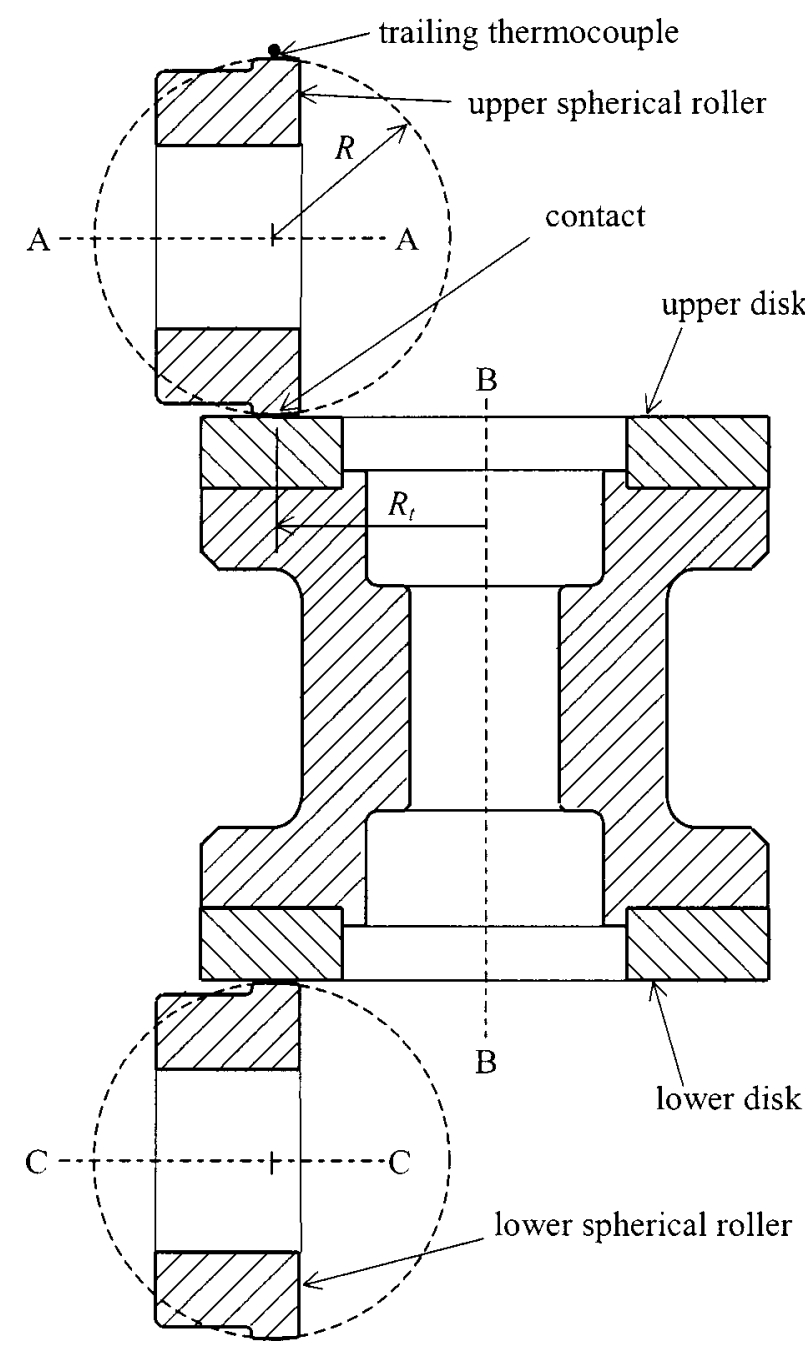

Fig. 1 Test head arrangement of the Torotrak traction rig with contact between spherical rollers of radius $R$ rotating about axes AA and CC, and plane disks, at a track radius $R_{t}$, rotating about axis BB

system. Unlike conventional two-disc rigs, a third cylindrical disc is introduced between the two spherical rollers. This component is in the form of a "bobbin" that is free to rotate about axis BB which is perpendicular to, and intersects, axes AA and CC. Two disk surfaces are incorporated in the bobbin, each in loaded contact with one of the spherical rollers. The purpose of the bobbin component is to introduce spin into the contact and thereby simulate the variator kinematics more closely. All test components used in the current work have polished surfaces of $R_{a}$ $<0.02 \mu \mathrm{m}$.

The spherical rollers are mounted on shafts supported by bearing housings. The lower bearing housing is restrained, and the upper bearing housing is mounted using long parallel rods terminated in ball joints that constrain any motion of the housing so that AA remains parallel to $\mathrm{CC}$. The traction force at the top contact is measured using a load cell which restrains the top roller bearing housing, and the contact normal load is applied by calibrated dead-weights, also applied to the top roller housing. The traction force at the bottom roller is not measured in the current work. 


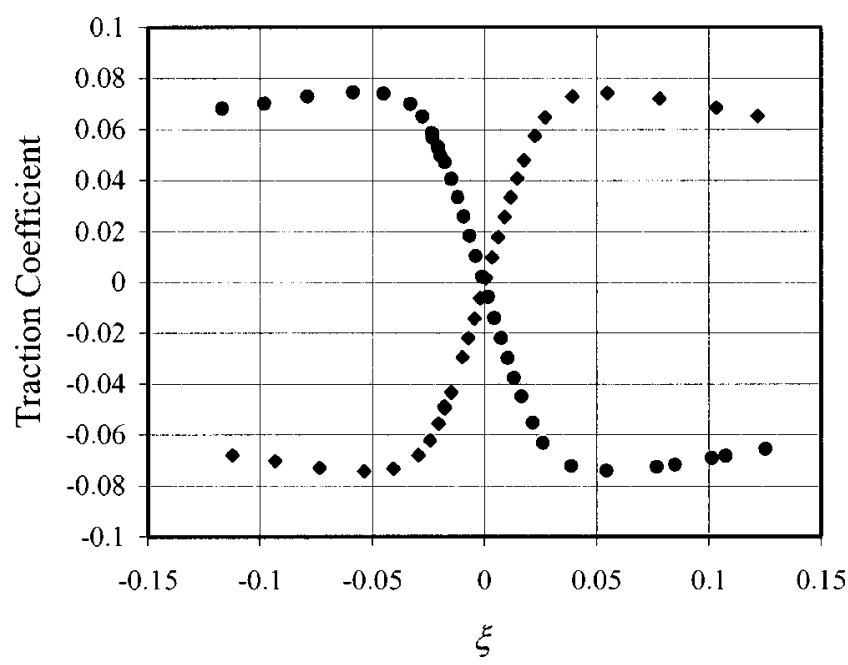

Fig. 2 Processed data from traction test ( clockwise rotation, anticlockwise rotation) of top shaft

Table 1 Test conditions used for full range of traction tests giving 54 tests in total

\begin{tabular}{llll}
\hline \hline \multicolumn{1}{c}{ Hertzian pressure } & $1 \mathrm{GPa}$ & $2 \mathrm{GPa}$ & $3 \mathrm{GPa}$ \\
\hline Oil supply temperature & $60^{\circ} \mathrm{C}$ & $90^{\circ} \mathrm{C}$ & $120^{\circ} \mathrm{C}$ \\
Entrainment velocity & $4 \mathrm{~m} / \mathrm{s}$ & $11 \mathrm{~m} / \mathrm{s}$ & $18 \mathrm{~m} / \mathrm{s}$ \\
Disk track radius & \multicolumn{2}{c}{$30 \mathrm{~mm}$} & $47 \mathrm{~mm}$ \\
\hline \hline
\end{tabular}

Since the speed of the intermediate flat disc is dependent on the speeds of the upper and lower rollers and the level of slip in the upper and lower contacts, an additional pre-load is applied to the lower contact through the bobbin shaft using a thrust bearing and pneumatic cylinder. This ensures that the surface speed of the bobbin is closely matched to that of the bottom roller so that the slip at the upper contact is more easily controlled through control of the speeds of the top and bottom rollers. The speeds of the rollers and bobbin are measured using optical encoders with a resolution of 360 pulses per revolution.

The test lubricant is maintained at the desired test temperature through PID control of a chilled water supply to a lubricant heat exchanger. Approximately 3 litres per minute of lubricant is supplied to each of the contacts through two jets, positioned either side of the contact such that similar levels of lubricant are supplied to the contact in both rotational directions. Test roller temperatures are monitored throughout the test using trailing thermocouples, positioned on the roller running track at an angular position that is $180^{\circ}$ from the traction contact.

During a standard traction test four individual traction curves are generated by running the rig in both the clockwise and anticlockwise directions and also by running both the top and bottom shafts as the driver during the test. An example of these traction curves is shown in Fig. 2. Each of the traction curves may then be compared to check the repeatability of the test. This test methodology has been adopted to ensure that any residual forces, perhaps due to dynamic loads or due to poor alignment of the contact normal load are eliminated in the test results.

The full range of conditions considered in the experimental program for any given materials/fluid combination is specified in Table 1 . The spherical roller radius is $R=30 \mathrm{~mm}$ so that for steel contacting components the Hertzian contact area has diameters of $0.85,1.71$, and $2.57 \mathrm{~mm}$ for the three loads utilized.

For the modeling work reported in this paper traction curves for

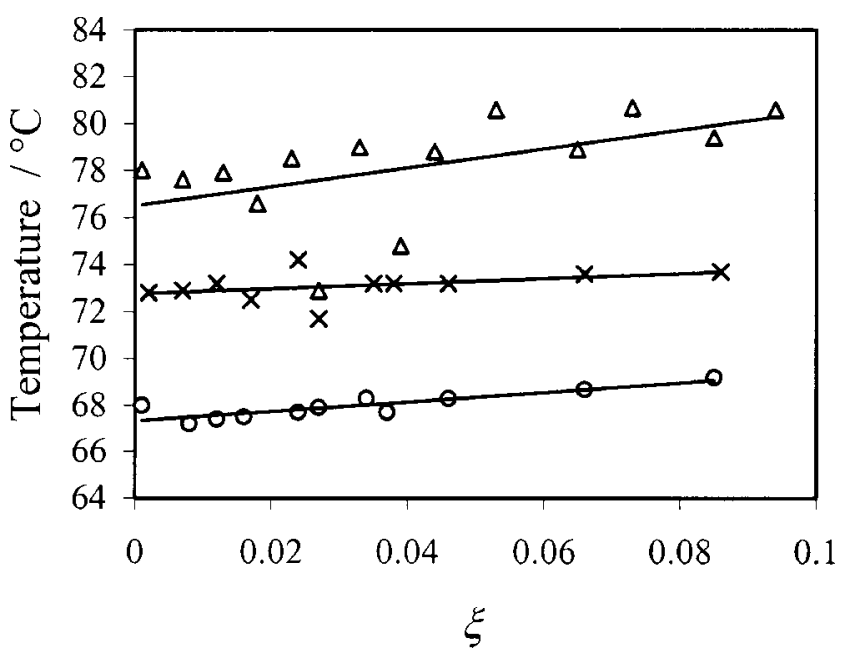

Fig. 3 Variation of test track thermocouple temperature for the tests at $60^{\circ} \mathrm{C}: \bigcirc \hat{u}=4 \mathrm{~m} / \mathrm{s}, \times \hat{u}=11 \mathrm{~m} / \mathrm{s}, \square \hat{u}=18 \mathrm{~m} / \mathrm{s}$

Table 2 Coefficients in the bulk temperature formula, $\theta_{B}$ $=\theta_{B 0}+\gamma \xi$ for the traction experiments

\begin{tabular}{cccc}
\hline \hline$\theta_{\text {ref }} /{ }^{\circ} \mathrm{C}$ & $\hat{u} /(\mathrm{m} / \mathrm{s})$ & $\theta_{B 0} /{ }^{\circ} \mathrm{C}$ & $\gamma /{ }^{\circ} \mathrm{C}$ \\
\hline 60 & 4 & 67.3 & 20.5 \\
& 11 & 72.8 & 10.8 \\
90 & 18 & 76.5 & 40.4 \\
& 4 & 102.8 & 0.593 \\
& 11 & 105.5 & 5.21 \\
\multirow{2}{*}{120} & 18 & 111.1 & -4.17 \\
& 4 & 140.6 & 10.9 \\
& 11 & 141.2 & 28.1 \\
& 18 & 145.9 & 23.1 \\
\hline
\end{tabular}

the $1 \mathrm{GPa}$ load and $30 \mathrm{~mm}$ disk track radius were used for reasons discussed later. The temperature measurement from the trailing thermocouple was subject to some noise during a traction test as can be seen from the examples shown in Fig. 3. This information was incorporated into the traction modeling by fitting the temperature measurements with straight lines and using these lines to determine the component bulk temperature for any given slide/roll ratio for each experiment. These temperature formulas are given in Table 2 for the $1 \mathrm{GPa}$ experiments with $R_{t}=30 \mathrm{~mm}$.

Clearly the high speeds attained in the tests lead to significant inlet shear heating irrespective of the degree of slip, so that the bulk temperatures of the components are determined by the cooling mechanism of the traction fluid spray. Cooling was not closely controlled over the duration of the traction tests which accounts for the lack of systematic variation in the values of parameter $\gamma$ in Table 2. However, developments since these tests were carried out have involved the fitting of further thermocouples which give confidence that the recorded temperatures are a good measure of component bulk temperatures.

\section{EHL Theory}

The Reynolds equation for the analysis of a point contact where the global $x$-axis and the local sliding direction differ by an angle $\phi$ is [7] 


$$
\begin{aligned}
\frac{\partial}{\partial x}\{ & \left.\left(\sigma_{s} \cos ^{2} \phi+\sigma_{r} \sin ^{2} \phi\right) \frac{\partial p}{\partial x}\right\} \\
+ & \frac{\partial}{\partial y}\left\{\left(\sigma_{s} \sin ^{2} \phi+\sigma_{r} \cos ^{2} \phi\right) \frac{\partial p}{\partial y}\right\} \\
+ & \frac{\partial}{\partial x}\left\{\left(\sigma_{s}-\sigma_{r}\right) \cos \phi \sin \phi \frac{\partial p}{\partial y}\right\} \\
+ & \frac{\partial}{\partial y}\left\{\left(\sigma_{s}-\sigma_{r}\right) \cos \phi \sin \phi \frac{\partial p}{\partial x}\right\} \\
= & \frac{\partial}{\partial x}(\rho \hat{u} h)+\frac{\partial}{\partial y}(\rho \hat{v} h)
\end{aligned}
$$

This reduces to the conventional form

$$
\frac{\partial}{\partial x}\left\{\sigma_{s} \frac{\partial p}{\partial x}\right\}+\frac{\partial}{\partial y}\left\{\sigma_{r} \frac{\partial p}{\partial y}\right\}=\frac{\partial}{\partial x}(\rho \hat{u} h)+\frac{\partial}{\partial y}(\rho \hat{v} h)
$$

for the special case $\phi=0$ where the sliding direction is in the $x$ direction at all points, and also for Newtonian situations where the flow factors $\sigma_{s}$ and $\sigma_{r}$ are identical. Equation (1) describes the hydrodynamic aspects of the EHL mechanism. The flow factors $\sigma_{s}(h, p, \partial p / \partial s, \partial p / \partial r)$ and $\sigma_{r}(h, p, \partial p / \partial s, \partial p / \partial r)$ are those in the sliding, $s$, and non-sliding, $r$, directions, and are obtained from the appropriate non-Newtonian rheology model as described in detail in reference [7]. The coupled nature of the shear stress/shear strain rate relationship when non-Newtonian lubricant rheology is specified does not lead to explicit functions for $\sigma_{s}$ and $\sigma_{r}$. Their values are determined by integrating the shear strain rate components (in the sliding, $s$, and nonsliding, $r$, directions) numerically across the film thickness. This numerical integration, although time consuming, can allow the effect of the cross film temperature variation on lubricant viscosity to be properly taken into account. This is an important consideration in seeking to model traction situations. Applying nonslip boundary conditions then enables the shear stress in these two directions to be established across the film. The shear stress at the surface is, of course, a key requirement in calculating the traction at the EHL conjunction. For some nonNewtonian rheological models, e.g., limiting shear stress models, the effective entrainment velocities in the axis directions, $\hat{u}$ and $\hat{v}$, can differ from the kinematic entrainment velocity components due to non-Newtonian effects as is also described in [7].

The elastic deflection is given by the deflection of contacting semi-infinite bodies, so that the film thickness is given by Eq. (2) in the form

$$
h(x, y)=h_{0}+\frac{x^{2}+y^{2}}{2 R}+\frac{2}{\pi E^{\prime}} \iint_{A} \frac{p\left(x^{\prime}, y^{\prime}\right)}{\sqrt{\left(x^{\prime}-x\right)^{2}+\left(y^{\prime}-y\right)^{2}}} d x^{\prime} d y^{\prime}
$$

This is incorporated into the solution scheme in differential form as developed by Holmes et al. [12] so that the equation is discretized as

$$
\frac{\partial^{2} h\left(x_{i}, y_{j}\right)}{\partial x^{2}}+\frac{\partial^{2} h\left(x_{i}, y_{j}\right)}{\partial y^{2}}=\frac{2}{R}+\frac{2}{\pi E^{\prime}} \sum_{\text {all } k, l} f_{k-i, l-j} p_{k, l}
$$

The weighting functions, $f_{i, j}$, for the influence of pressure on the deflection Laplacian, are evaluated as described in [13]. The rapid decay of these weighting functions with increasing index allows Eqs. (1) and (3) to be solved as a coupled pair as described in [12].

To deal with the sliding situation the energy equation for the fluid is given by

$$
\begin{aligned}
\rho c\left(u \frac{\partial \theta}{\partial x}+v \frac{\partial \theta}{\partial y}\right)= & \tau_{x} \frac{\partial u}{\partial z}+\tau_{y} \frac{\partial v}{\partial z}+\varepsilon \theta\left(u \frac{\partial p}{\partial x}+v \frac{\partial p}{\partial y}\right) \\
& +\frac{\partial}{\partial x}\left(k \frac{\partial \theta}{\partial x}\right)+\frac{\partial}{\partial y}\left(k \frac{\partial \theta}{\partial y}\right)+k\left(\frac{\partial^{2} \theta}{\partial z^{2}}\right)
\end{aligned}
$$

The boundary conditions for this equation are the surface temperatures of the contacting components. These are obtained using a simple one-dimensional (linear heat flow) conduction model so that the surface temperatures are given by integrals of the form

$$
\theta_{S}=\theta_{B}+\frac{1}{\sqrt{\pi k \rho c}} \int_{0}^{t} \frac{q d \lambda}{\sqrt{t-\lambda}}
$$

where, for the steady state problem under consideration, the time integration becomes a spatial integration along the component's locus through the contact area.

\section{Solution Method}

The method by which the equations are solved is presented in detail in references [7] and [12] and is given in outline in the current paper for completeness. The innermost loop of the EHL solution method consists of the simultaneous solution of Eqs. (1) and (3). The way in which the elastic equation has been posed in differential form together with the rapid decay of the weighting functions $f_{i, j}$ as the indices $i$ and $j$ increase from zero [13] allows the equations to be solved simultaneously with a simple iterative method.

Equation (1) is discretised using a Finite Elements (FE) method with linear elements. When assembled into an overall FE problem the equations for each node involve the pressure and film thickness values at the node and its eight surrounding neighboring nodes. Thus, assembled Eqs. (1) for the $(i, j)$ node can be written in the form

$$
\sum_{k=0}^{n_{c}} A_{k} p_{k}+\sum_{k=0}^{n_{c}} B_{k} h_{k}=R_{i, j}
$$

where suffix $k$ represents the nodes contributing to the assembled equation at node $(i, j)$ and $k=0$ denotes that node. $A_{k}$ and $B_{k}$ are the pressure and film variable coefficients for the Reynolds equation, and $n_{c}$ is the number of neighboring nodes involved in the formulation. The method does not depend on an FE approach for its success and detailed comparisons between the FE formulation and the corresponding treatment where the Reynolds Equation is discretised using a central difference formulation are discussed in [12].

The left hand side of Eq. (3) is the Laplacian of film thickness, and is discretised using central differences. The pressure summation on the right hand side is partitioned into those terms that involve the pressure at node $(i, j)$ and its $n_{c}$ neighbors, which are moved to the left hand side, and the remainder which are retained on the right hand side. Equation (3) can then be written in a discretized form corresponding to Eq. (6) as

$$
\sum_{k=0}^{n_{c}} C_{k} p_{k}+\sum_{k=0}^{n_{c}} D_{k} h_{k}=E_{i, j}
$$

where $C_{k}$ and $D_{k}$ are the pressure and film variable coefficients for the differential deflection equation. (The use of central differences for the Laplacian of $h$ gives $D_{k}$ coefficients that are zero for the neighboring nodes diagonal to node $(i, j))$. The right hand side, $E_{i, j}$, contains the pressure summation $\Sigma_{\text {all } k, l} f_{k-i, l-j} p_{k, l}$ for all pressure contributions except those incorporated in the first term of the left hand side. The summation contributing to $E_{i, j}$ is split into two parts as $\sum_{\text {close }} f_{k-i, l-j} p_{k, l}+\sum_{\text {far }} f_{k-i, l-j} p_{k, l}$ so that at each point in the mesh there are three regions contributing to the pressure summation. The near region is that embodied in the left hand side of Eq. (7), which corresponds to the point at which the equation is applied and its $n_{c}$ nearest neighbors $(i \pm 1, i \pm 1)$. The close 
region is a band of mesh points surrounding the near region, and the far region makes up the remainder of the summation. The differential deflection formulation results in pressure weighting coefficients whose magnitudes fall rapidly to zero as their indices increase [13]. The contribution to $E_{i, j}$ from close and far contributions can be linearized as a result so that the coupled equations to be solved are Eqs. (6) and (7). The equations are solved by an iterative method. Simple Gauss Seidel point iteration where Eq. (6) is used as an iterative modification for pressure at node $(i, j)$ and Eq. (7) as an iterative modification for film thickness was found to be unstable and unsuitable. Instead Eqs. (6) and (7) are organized in the form

$$
\begin{aligned}
& A_{0} p_{0}+B_{0} h_{0}=\hat{R}_{i, j}\left\{=R_{i, j}-\sum_{k=l}^{n_{c}} A_{k} p_{k}-\sum_{k=l}^{n_{c}} B_{k} h_{k}\right\} \\
& C_{0} p_{0}+D_{0} h_{0}=\hat{E}_{i, j}\left\{=E_{i, j}-\sum_{k=l}^{n_{c}} C_{k} p_{k}-\sum_{k=l}^{n_{c}} D_{k} h_{k}\right\}
\end{aligned}
$$

which are regarded as a pair of iterative equations to be solved simultaneously for the updated values of $p_{0}$ and $h_{0}$ (i.e., the nodal values at node $(i, j))$. The new iterative values at the node are thus

$$
\left.\begin{array}{l}
p_{i, j}^{\text {new }}=\frac{\hat{R}_{i, j} D_{0}-\hat{E}_{i, j} B_{0}}{A_{0} D_{0}-B_{0} C_{0}} \\
h_{i, j}^{\text {new }}=\frac{\hat{E}_{i, j} A_{0}-\hat{R}_{i, j} C_{0}}{A_{0} D_{0}-B_{0} C_{0}}
\end{array}\right\}
$$

and simple iteration using this pair of expressions is found to solve the coupled equations rapidly. Under-relaxation is not generally required for smooth surface problems. The boundary of the "close" region is square and typically encloses 289 mesh points for equal mesh sizes $\Delta x$ and $\Delta y$. For cases with differing values of $\Delta x$ and $\Delta y$ it can be advantageous to extend the "close" region boundaries in the finer mesh direction so that the points included in the close region lie within a square area centred on the point $(i, j)$. The coefficients $A_{k}$ and $B_{k}$ and the "close" contribution to $E_{i, j}$ are re-calculated at the end of each iterative solution of Eqs. (1) and (3). The "far" contribution is re-calculated periodically as required [12].

The boundary equations to be specified for the Reynolds equation are that pressure is everywhere positive, and fixed at zero on the boundaries of the computing region. The boundary conditions required for Eq. (3) are values of $h$ on the boundaries of the computing region. These are obtained by applying Eq. (2) using the pressure distribution from the outer loop of the current timestep, with the deflection on the boundary obtained from a discretized form of the conventional integral equation for deflection. Equation (8) is thus used to modify the pressure and film thickness fields iteratively subject to these boundary conditions.

The thermal problem is solved in an outer loop so that as the EHL solution of equations (1) and (3) is obtained, the temperature distribution within the film becomes established. Thus the temperature dependence of viscosity is taken into account in determining the flow coefficients $\sigma_{r}$ and $\sigma_{s}$. In solving Eq. (4) numerically the film is partitioned into $n_{f}$ cross film node points. The right hand side and the velocity and pressure gradient dependent coefficients in the terms in $\theta$ and its derivatives are evaluated at each cross film node point using the outer loop values of these parameters. The conductive derivative terms are expressed in central difference form, and backward or forward differences are used for the convective terms according to the sign of the fluid velocity components at each mesh point and level. The current values of the surface temperatures are regarded as boundary conditions and thus there are $n_{f}-2$ equations in the $n_{f}-2$ cross film node point temperatures at each $(x, y)$ position. The temperature values at other $(x, y)$ positions are taken as their current approximation (outer loop) values. Thus at each $(x, y)$ position there are $n_{f}-2$ equations in $n_{f}-2$ unknowns. These equations are solved with a tridiagonal solver to produce a new cross film temperature field. Temperature boundary conditions are imposed at the boundary at all $z$ values where oil is flowing in to the computing region. The equation is not solved on the boundary but at points adjacent to the boundary. At boundary positions and $z$ values where the oil is flowing out of the computing region the treatment of the convective terms ensures that no boundary condition is imposed through these terms. The second order conductive terms require a boundary condition to be imposed and for outflowing lubricant this is achieved by specifying that there is no heat flux out of the computing region.

To complete the temperature calculation the temperature gradient, $\partial \theta / \partial z$, is evaluated at the solid/liquid interfaces and used to give the surface heat flux, $q$, so that each of the surface temperatures may be re-calculated from Eq. (5). For each point on the surface the integral of Eq. (5) is evaluated taking note of the locus of the surface point in reaching its current position so that the time integral is converted into a spatial integral over a curved path determined by the motion of the component relative to the instantaneous contact point. Each body's reference surface temperature is taken as the bulk temperature value for the component. In this way each of the two solid bodies is assumed to enter the computing region at the specified (possibly different) bulk temperature for that component, and thus the thermal model allows the appropriate surface flash temperatures to be calculated.

This sequence of thermal calculations is carried out once for each cycle of the EHL convergence process. The interface temperature gradients and cross film temperature distribution are found to stabilize quickly and converge reliably. The overall solution is obtained when the pressure, film thickness and temperature fields converge with the constant $h_{0}$ in the film thickness equation adjusted to obtain the required load.

\section{Lubricant Rheology}

The traction experiments modeled in this work were conducted using Santotrac 50, a commercial traction fluid. This fluid was selected because its rheological properties have been the focus of several investigations reported in the literature $[9,14]$. In particular Bair and Winer [9] have obtained viscosity/temperature/pressure data for Santotrac 50 and have fitted their experimental viscosity measurements for this fluid to the Yasutomi equation [15]:

$$
\eta=\eta_{g} \exp \left\{\frac{-2.3 C_{1}\left(T-T_{g}\right) F}{C_{2}+\left(T-T_{g}\right) F}\right\}
$$

where

$$
T_{g}=T_{g 0}+A_{1} \ln \left(1+A_{2} p\right)
$$

and

$$
F=1-B_{1} \ln \left(1+B_{2} p\right)
$$

Values for the constants $A_{1}, A_{2}, B_{1}, B_{2}, C_{1}, C_{2}, T_{g 0}$, and $\eta_{g}$ for Santotrac 50 are given [9] and reproduced in Table 3. The viscosity behavior described by this particular form of Eq. (9) is illustrated in Fig. 4.

The data used for obtaining the fit [9] were at temperatures of $70^{\circ} \mathrm{C}, 100^{\circ} \mathrm{C}$, and $140^{\circ} \mathrm{C}$ and up to maximum pressures of 0.8 $\mathrm{GPa}, 1 \mathrm{GPa}$, and $1.2 \mathrm{GPa}$, respectively. The viscosity isotherms that correspond to this data area are shown with thicker lines in Fig. 4 up to these maximum pressures. The form of the Yasutomi

Table 3 Values for the Yasutomi equation constants $A_{1}, A_{2}$, $B_{1}, B_{2}, C_{1}, C_{2}, T_{g 0}$, and $\eta_{g}$ for Santotrac 50 given in [9]

\begin{tabular}{cccc}
\hline \hline$A_{1} /{ }^{\circ} \mathrm{C}$ & $A_{2} / \mathrm{GPa}^{-1}$ & $B_{1}$ & $B_{2} / \mathrm{GPa}^{-1}$ \\
\hline 92.92 & 2.600 & 0.2965 & 16.275 \\
\hline \hline$C_{1}$ & $C_{2} /{ }^{\circ} \mathrm{C}$ & $T_{g 0} /{ }^{\circ} \mathrm{C}$ & $\eta_{g} /$ Pa.s \\
\hline 10.51 & 20.70 & -46.9 & $10^{7}$ \\
\hline \hline
\end{tabular}




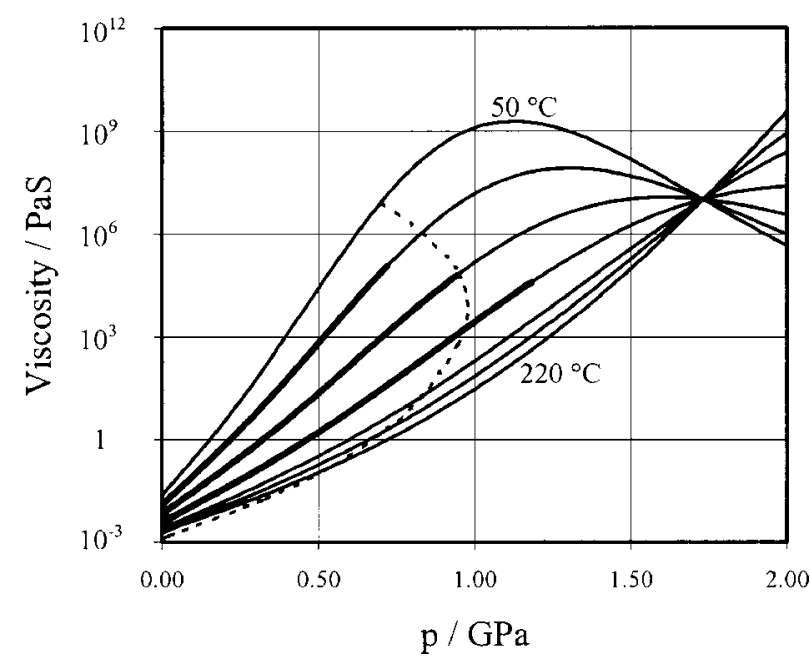

Fig. 4 Yasutomi viscosity formula for Santotrac 50 [16] for temperatures of $50,70,100,140,180,200$, and $220^{\circ} \mathrm{C}$

equation constrains the curves to pass through the common point $\eta=\eta_{g}$ at the pressure value that makes $F=0$, which in this case is $1.73 \mathrm{GPa}$. Although the formula provides an excellent fit to the viscosity measurements over the range of the data it clearly cannot be used generally to extrapolate outside this region as, for example, at the $2 \mathrm{GPa}$ level it shows viscosity to be increasing with temperature. The Yasutomi formula was used in a curtailed form in two ways. First, outside the area limited by the broken curve shown in Fig. 4 extrapolation was according to tangents to the viscosity isotherms at this boundary, denoted Model I. Second, the low pressure viscosity/pressure behavior was used to extrapolate to all higher pressures, denoted Model II. Model II clearly discounts the greater than exponential viscosity pressure variation measured by Bair and Winer but has the attraction that the low pressure viscosity behavior can be obtained from EHL film thickness measurements. These two models can be regarded as extremes of possible viscosity/pressure extrapolation. However, to avoid conclusions based upon gross extrapolation of known viscosity pressure data, traction modelling described in this paper was concentrated on the $1 \mathrm{GPa}$ experimental cases which remained in or close to Bair and Winer's experimental data area. The intention was to establish an appropriate rheological model at this pressure level, and to use data from higher pressure levels subsequently to clarify appropriate pressure viscosity extrapolations.

The non-Newtonian behavior of the lubricant was modelled taking the shear strain rate to be a nonlinear function $F(\tau)$ of the shear stress $\tau$. Different non-linear relationships were used during the study based on the models of Johnson and Tevaarwerk [4], Bair and Winer [8], and Sharif et al. [7] according to Eqs. (10), (11), and (12) respectively as follows:

$$
\begin{gathered}
F(\tau)=\frac{\tau_{0}}{\eta} \sinh \left(\frac{\tau}{\tau_{0}}\right) \\
F(\tau)=-\frac{\tau_{L}}{\eta} \ln \left(1-\tau / \tau_{L}\right) \\
F(\tau)=\frac{\tau_{0}}{\eta} \sinh \left(\frac{\tau}{\tau_{0}}\right)\left\{\frac{\tau_{L}^{2 \nu}}{\tau_{L}^{2 \nu}-\tau^{2 \nu}}\right\}
\end{gathered}
$$

The first of these models exhibits so-called Eyring behavior, the second, limiting shear stress behavior, and the third a combination of both. These formulations were used to determine the flow coefficients $\sigma_{s}$ and $\sigma_{r}$ in Eq. (1), and the shear stresses $\tau_{x}$ and $\tau_{y}$ in equation (4) throughout the film as described in [7].

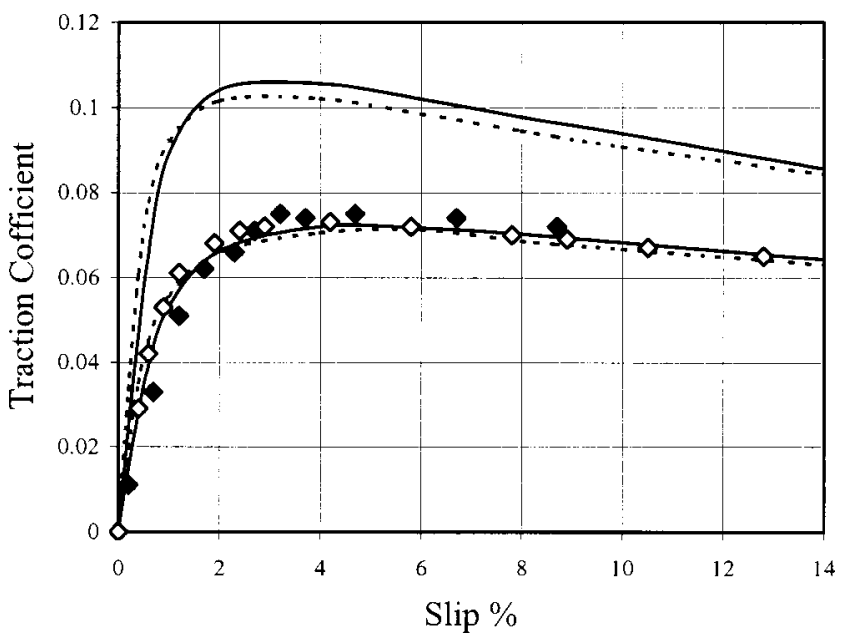

Fig. 5 Traction curves obtained using an Eyring model for the $90^{\circ} \mathrm{C}, 11 \mathrm{~m} / \mathrm{s}$ experiments. Viscosity Model I upper curves, Model II lower curves, symbols show experiments. Solid curves and $\diamond, R_{t}=30 \mathrm{~mm}$; broken curves and $\diamond, R_{t}=47 \mathrm{~mm}$.

\section{Traction Modelling}

Initial modeling of the traction experiments was carried out using the Eyring model of Eq. (10). Values of parameter $\tau_{0}$ derived from traction experiments given by Evans and Johnson [16] show the parameter to be pressure and temperature dependent. These data can be seen to vary linearly with temperature and pressure according to

$$
\tau_{0}=5.62 \times 10^{6}+2.54 \times 10^{4} T-2.17 \times 10^{-3} p
$$

where $T$ is the temperature in ${ }^{\circ} \mathrm{C}$ and $p$ is the pressure in $\mathrm{Pa}$. The EHL problem was solved for a range of sliding speeds for each of the current traction experiments specifying $\tau_{0}$ in this way everywhere within the film. The mesh spacing adopted for the modelling had $\Delta x=a / 60$ and $\Delta y=a / 60$, and a typical computational area of $-1.5 a<x<1.5 a,-1.5 a<y<1.5 a$. This was extended as necessary to avoid starvation effects. For the thermal analysis the number of cross film levels was $n_{f}=7$. Figure 5 compares traction curves obtained using the Eyring non-Newtonian model for one particular traction experiment. Results are shown using both Model I and Model II for the viscosity/pressure behavior. The difference between these traction curves emphasizes the sensitivity of any such calculation to the viscosity/pressure behavior assumed. The critical behavior as far as traction drive modelling is concerned is the low slip region; the magnitude of the maximum traction coefficient; and the value of slip at which it occurs (corresponding to "runaway"). The measurements of $\tau_{0}$ on which equation (13) is based, although indicating dependence on pressure and temperature, were derived based on an average temperature and assumed film thickness and pressure distributions [16]. It had been anticipated that this $\tau_{0}$ behavior would give a good match to the measured traction behavior over the range of experiments: however this was found not to be the case. The results shown in Figure 5 include both the $R_{t}=30 \mathrm{~mm}$ and $R_{t}=47 \mathrm{~mm}$ cases to show the effect of reducing the relative effect of spin at the contact. The change in traction behavior brought about by this change in track radius can be seen to be small both from the experimental and modelling viewpoint. The small differences observed experimentally of higher initial slope and lower final traction values for the $R_{t}=47 \mathrm{~mm}$ case were also generally replicated in the numerical modelling for a given rheological model. The experience obtained suggests strongly that models which follow the experimental data for the $R_{t}=30 \mathrm{~mm}$ case will also follow the experimental data for the corresponding $R_{t}=47 \mathrm{~mm}$ case. Conse- 

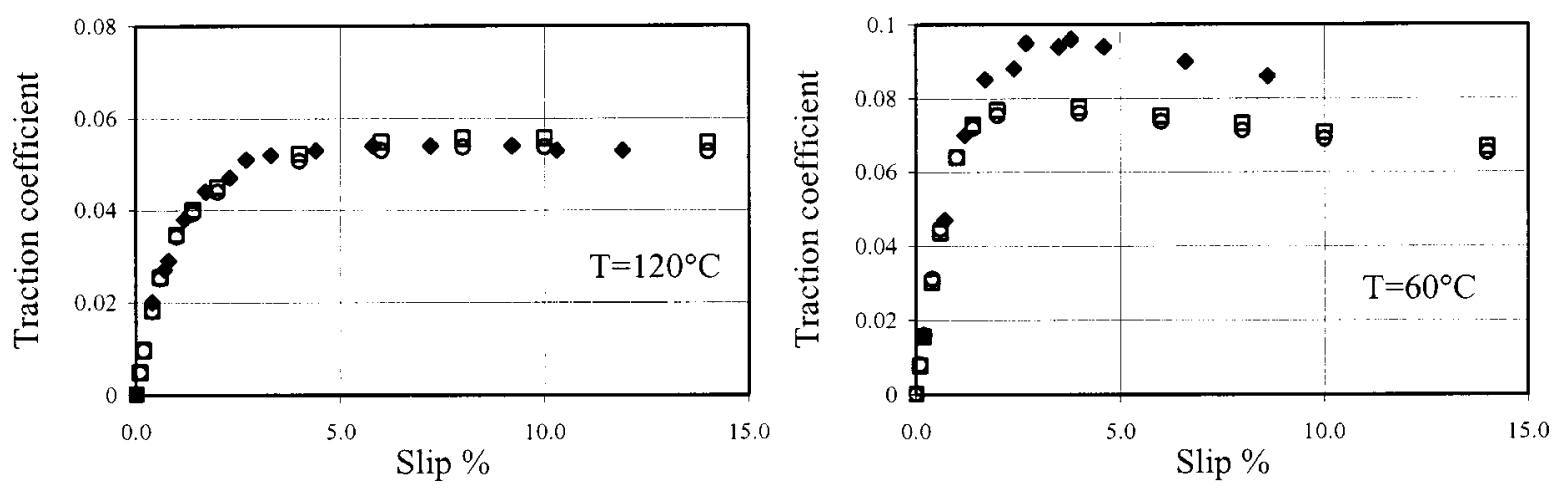

Fig. 6 Traction curves at two temperatures with $11 \mathrm{~m} / \mathrm{s}$ entrainment velocity using $\tau_{0}=A$ and $\tau_{0}=B p$ established from low slip behavior; $\bullet$ experiment, $\square \tau_{0}=A, \bigcirc \tau_{0}=B p$

quently the modelling effort concentrated on the $R_{t}=30 \mathrm{~mm}$ experiments as these had the higher degree of spin.

The component bulk temperatures in the experiments were not known with precision but a good indication of their temperature was given by a trailing thermocouple measurement which was taken on the opposite side of the roller component as described above (see Fig. 1). For each experiment the trailing thermocouple temperature value was plotted against the sliding velocity and a straight line placed through these points as shown in Fig. 3, for example. At any given sliding speed the bulk temperature of the contacting components at entry to the computing region was taken from this straight line. This was an important adjustment as the component temperatures increased by as much as $28^{\circ} \mathrm{C}$ above the oil supply temperature as sliding increased and the procedure was used for all the traction results obtained by numerical modeling. To assess the temperature dependence of $\tau_{0}$ the low slip behavior of each experiment was analyzed so as to minimize film and component temperature rise. Full EHL thermal Eyring models were developed for the experimental results in two forms: (i) where $\tau_{0}=A$, and (ii) where $\tau_{0}=B p$. For each experiment the values of constants $A$ and $B$ were obtained so as to match the low slip traction behavior.

For the nine $30 \mathrm{~mm}$ track radius traction curves optimum values of $A$ and $B$ for the low slip region were established in each case, as shown in Fig. 6, for example. For the high temperature experimental curves this gave a good fit to the three measured curves as shown in Fig. 6(a), but at the lower temperature the predicted traction falls off before the experimental values reach their peak as seen in Fig. 6(b). The model traction curves for the two cases ( $\tau_{0}=A$ or $\tau_{0}=B p$ ) were found to be very similar when the low slip behavior is used to establish the constants. Similar fits were also obtained using viscosity Model II, but at $120^{\circ} \mathrm{C}$ it was found that even with $\tau_{0}$ set so high as to ensure Newtonian behavior, the model could not generate sufficient shear stress to give the observed low slip traction behavior. This result confirms, first, the importance of the greater-than-exponential increase of viscosity with pressure given by Model I, to which Bair has consistently drawn attention, and second, that the low shear rate viscosity behavior cannot be used to successfully model traction with traction fluids that display this characteristic. Consequently Model I was used for viscosity/pressure dependence for the remainder of the investigation.

The values of the constants $A$ and $B$ needed to give these low slip fits are temperature-dependent as illustrated in Fig. 7 where the mean values at each temperature are also plotted. The variation of both constants with temperature follows a very similar pattern. A simple empirical temperature dependence, $q(T)$, of the form of the solid curves in Fig. 7 was determined and the full EHL traction models repeated in the form of either $\tau_{0}=A q(T)$ or $\tau_{0}$ $=B p q(T)$ with the constants $A$ and $B$ again chosen so as to match the low slip behavior. The results obtained using these tem- perature dependent models for $\tau_{0}$ are quite different, in their high slip behavior, to those described above and illustrated in Figure 6. An example of this is given in Fig. 8, where both the original and temperature dependent fits are shown. Inclusion of temperature dependence in this way raises the predicted traction, as expected, but removes the peak and subsequent decline that is characteristic of the experiments. Again, the two models (with and without pressure dependence) behave in a similar way but still fail to predict the peak traction behavior in detail.

Behavior at high slip is strongly affected by thermal response,

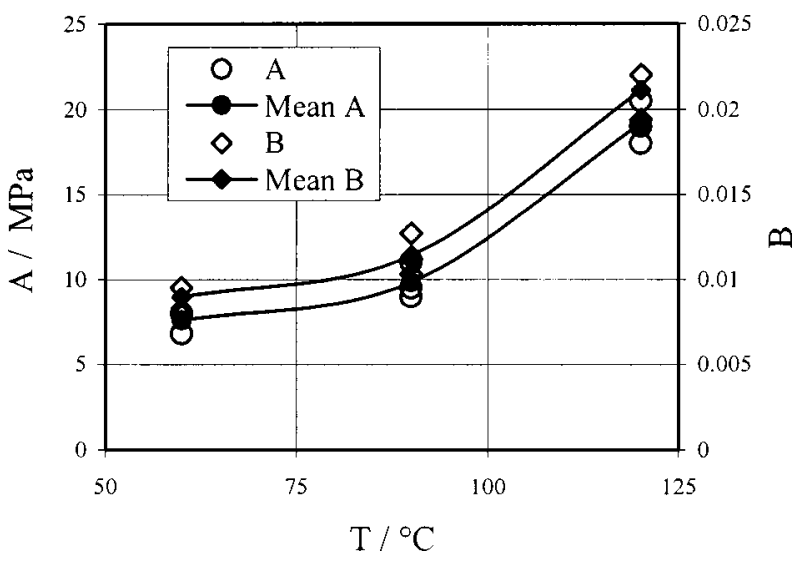

Fig. 7 Variation of constants $A$ and $B$ with nominal temperature of experiment

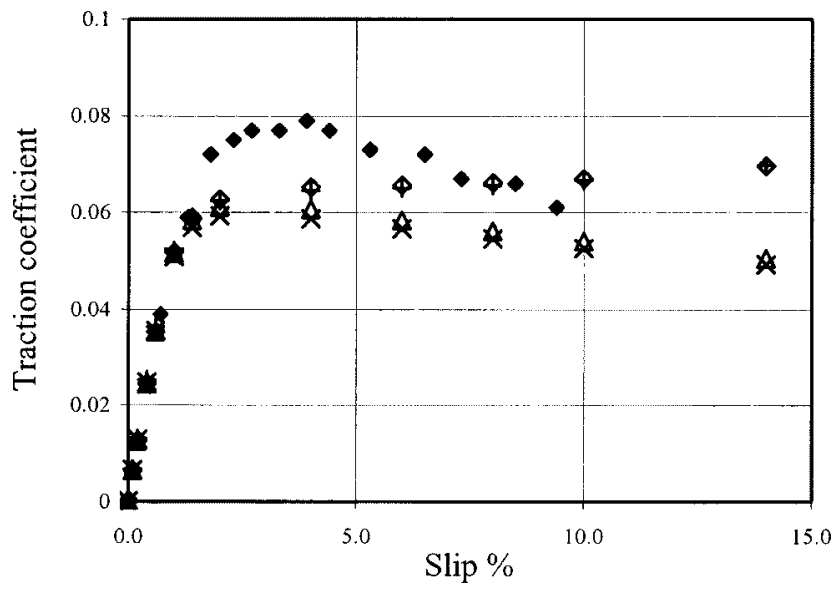

Fig. 8 Traction curves for $T=60^{\circ} \mathrm{C}, \hat{u}=18 \mathrm{~m} / \mathrm{s} ; \diamond \tau_{0}=A q(T)$, $+\tau_{0}=B p q(T), \square \tau_{0}=A, \times \tau_{0}=B p$, experiment 
Table 4 Best-fit coefficients established from low slip part of experimental traction curves using Eyring shear stress $\tau_{0}=A+B T$ and Limiting shear stress $\tau_{L}=(C+D T) p$

\begin{tabular}{lccccc}
\hline \hline & $\begin{array}{c}\text { Temperatures } \\
\text { Included/ }{ }^{\circ} \mathrm{C}\end{array}$ & $A / \mathrm{MPa}$ & $B / \mathrm{MPa}^{\circ} C^{-1}$ & $C$ & $D /{ }^{\circ} C^{-1}$ \\
\hline Equation (10) & $60,90,120$ & -15 & 0.135 & & \\
Equation (11) & $60,90,120$ & & & 0.1056 & $-1.873 \times 10^{-4}$ \\
Equation (12) & $60,90,120$ & -1.44 & 0.146 & 0.982 & $-1.1 \times 10^{-5}$ \\
Equation (10) & 90,120 & -8.0 & 0.18 & 0.084 & $-2.5 \times 10^{-5}$ \\
Equation (11) & 90,120 & & & 0.084 \\
\hline \hline
\end{tabular}

so the sensitivity of the model calculations to the values of the thermal property parameters was assessed. It was thought that a higher value of the oil thermal conductivity could lead to a smaller temperature rise with a consequential increase in the traction peak value. Tests of this hypothesis, however, indicate that the peak is not particularly sensitive to the thermal conductivity, with changes of $100 \%$ in its value resulting in only a 14\% change in the peak traction value. The failure to model the peak traction closely would thus seem to be a consequence of the way in which the parameter $\tau_{0}$ is modelled as a function of temperature and pressure, and not of the thermal conductivity which was obtained from [14].
It was thus decided to establish $\tau_{0}(p, T)=A+B T$ by undertaking a least-squares fit to each of the nine $1.0 \mathrm{GPa}$ traction curves. This kind of linear dependence of $\tau_{0}$ on temperature can be observed in the measurements of $\tau_{0}$ for Santotrac 50 given in [16]. Although the traction experiments available typically cover a range of up to $15 \%$ slip, the features of the behavior of key interest to traction drive design are confined to the range 0 to $4 \%$ slip. Experimental results at $1 \%, 2 \%$, and $4 \%$ slip were determined from the experimental curves, and the predicted values for these cases were obtained from the EHL model for each experimental condition for any trial model of $\tau_{0}$. A least-squares error measure based on the discrepancies between the 27 predicted and experi-
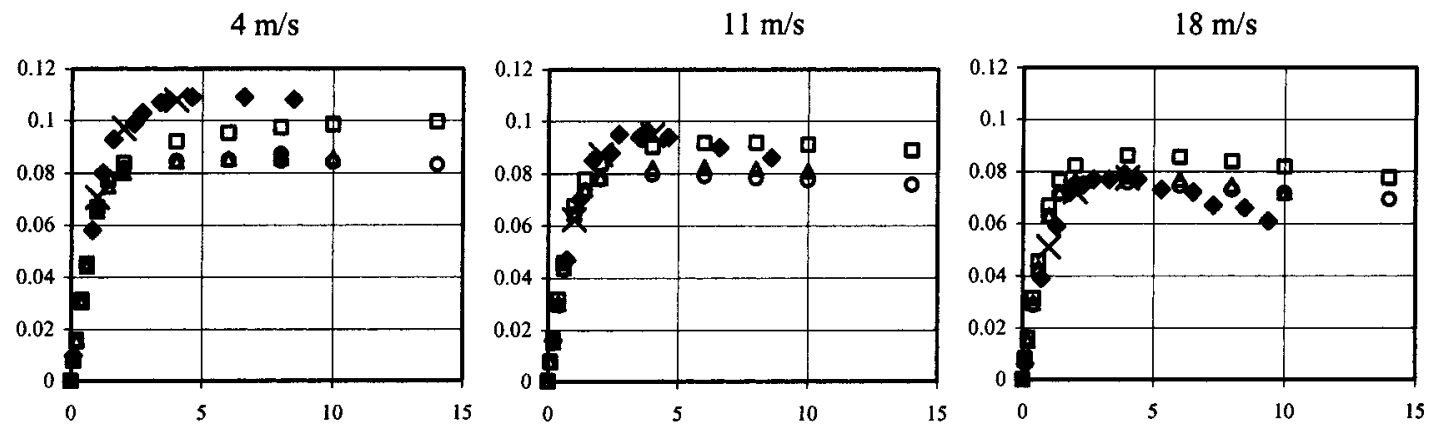

$60^{\circ} \mathrm{C}$
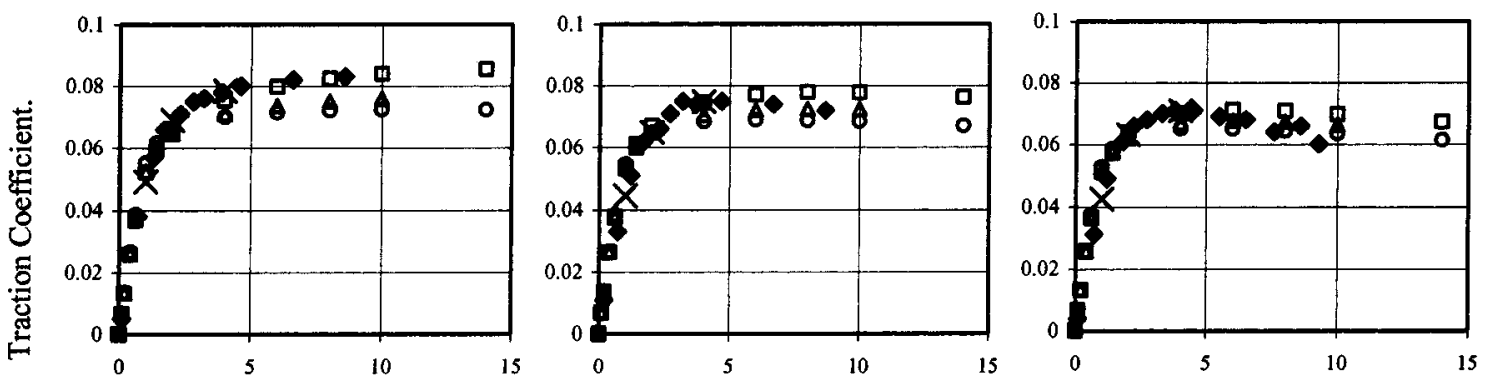

$90^{\circ} \mathrm{C}$
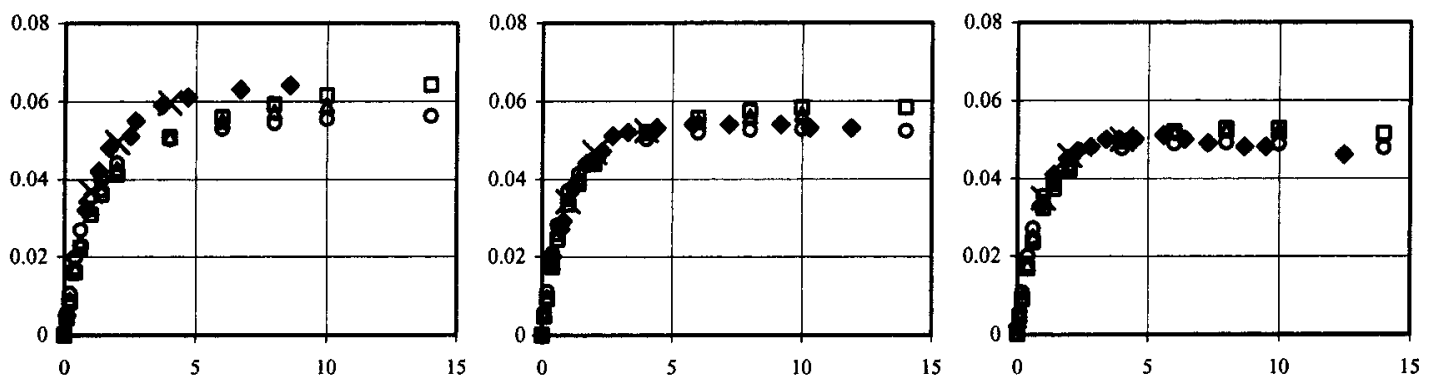

$120^{\circ} \mathrm{C}$

Slip \%

Fig. 9 Least square best fit models compared with experiment for $30 \mathrm{~mm}$ track radius experiments. $\downarrow$ experiment, $\square$ Eyring model, $\bigcirc$ limiting shear stress model, $\triangle$ combined model, $X$ points used for least square fit. Traction curves are arranged by column for entrainment speed and by row for experiment nominal temperature. 
$4 \mathrm{~m} / \mathrm{s}$
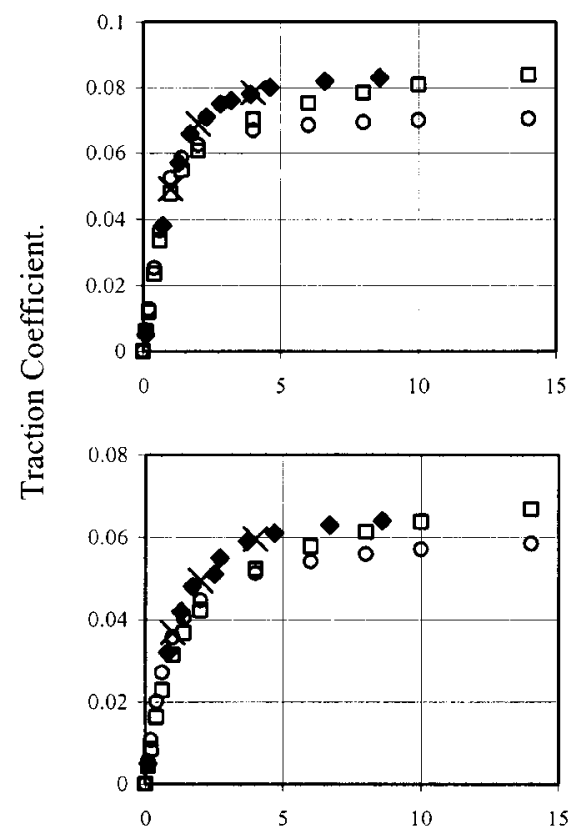

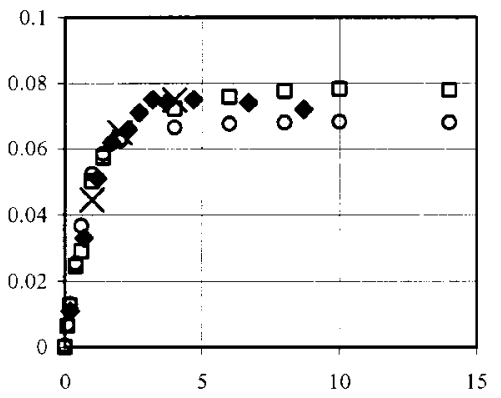

$11 \mathrm{~m} / \mathrm{s}$

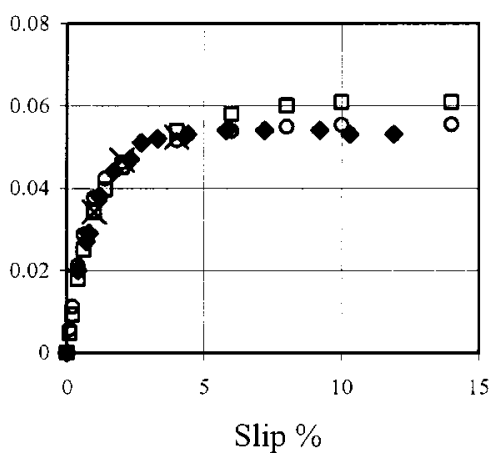

$18 \mathrm{~m} / \mathrm{s}$

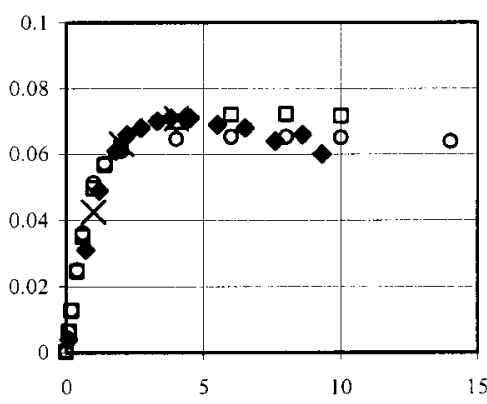

$90^{\circ} \mathrm{C}$

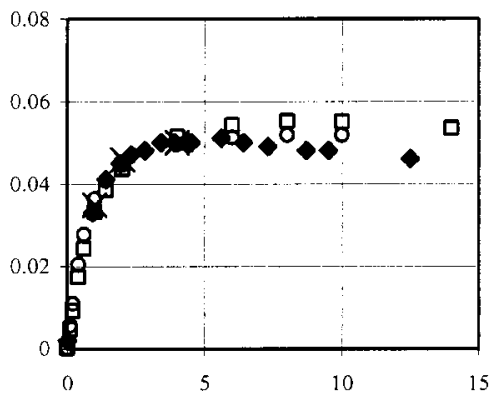

Fig. 10 Least square best fit models based on fits to the $90^{\circ} \mathrm{C}$ and $120^{\circ} \mathrm{C}$ experiments compared with experimental results. $\checkmark$ experiment, $\square$ Eyring model, $\bigcirc$ limiting shear stress model, $\times$ points used for least square fit. Traction curves are arranged by column for entrainment speed and by row for experiment nominal temperature.

mental traction values was then used to choose constants $A$ and $B$ so as to optimize the fit over the full range of experiments by minimizing this error. This best-fit approach was also used with the limiting shear stress model of Eq. (11) and with the combined model of Eq. (12). For Eq. (11) parameter $\tau_{L}$ was modeled as $\tau_{L}=(C+D T) p$ because measurements of limiting shear stress in the literature [17] have shown it to be proportional to pressure. For the combined model of Eq. (12) the two parameters $\tau_{0}$ and $\tau_{L}$ were modelled using $\tau_{0}=A+B T, \tau_{L}=(C+D T) p$, and parameter $\nu$ was chosen so as give a good approximation to the way in which Eq. (11) approaches the limiting shear stress value. This was achieved with a value of $\nu=3$. The model of equation (10) was also used with a further pressure dependence so that $\tau_{0}$ was modelled as $\tau_{0}(p, T)=A+B T+C p$. The fit obtained with $C$ set to zero was as good as those obtained with nonzero $C$ values indicating either that pressure dependence of $\tau_{0}$ is not significant, or that experimental results obtained at different load levels would have to be incorporated to enable the pressure dependence to be determined. The experimental results available were obtained at maximum Hertzian pressures of 1.0, 2.0, and 3.0 GPa, but the higher pressures took the viscosity far into the extrapolated region and so these higher load cases were not pursued.

\section{Discussion}

The particular values of the constants $A, B, C, D$, obtained from fitting the experimental data in this way are given in Table 4 . The best-fit traction curves for all three rheological models are shown in Fig. 9. The experimental points are shown as solid diamond symbols and the full thermal EHL model results with the three best-fit rheological models are included as open symbols. The three traction results at $1 \%, 2 \%$, and $4 \%$ slip that are the basis of the least-squares fitting process are shown as crosses. The most encouraging result from a best-fit point of view over this low slip range was obtained with the Eyring model. Beyond the $4 \%$ slip range the Eyring model traction curves are flat and do not show the drooping characteristic of the high-speed low-temperature experiments. The limiting shear stress best-fit models give lower traction at high slip but are less satisfactory at low slip. The combined model of Eq. (12), which has four parameters at its disposal to fit the experiments, was unable to improve on the fit of the Eyring model, which is interesting and unexpected. In general the agreement with the experimental behavior is best at high rolling speeds and high temperatures. The Eyring model, which gives the best overall fit, can be seen as a reasonably accurate working model up to the slip level of 5\% which characterizes safe traction drive behavior. Its deficiencies in this range are effectively a conservative evaluation of the available traction at low entrainment velocity. There is little difference between the two models that incorporate limiting shear stress behavior, indicating that the limiting shear strength is the key parameter in these models. None of the best-fit models exhibit the drooping nature of the experimental traction curves at low temperature. The less accurate fit at the lowest temperature of $60^{\circ} \mathrm{C}$ may be due to the greater viscosity/ temperature sensitivity at that temperature, and the relatively high viscosity. This may cause greater contact flash temperature rises in comparison with the higher-temperature experiments. Comparison of the value of parameter $B$ in Table 4 with the coefficient of temperature in Eq. (13) shows that the current work indicates a much stronger temperature dependence for $\tau_{0}$ than that reported by Evans and Johnson [16].

The temperature assumed for the analysis at each slip speed was taken from the trailing thermocouple measurement in the experiment and this may be a source of systematic error that is accentuated at $60^{\circ} \mathrm{C}$ where viscosity is much more sensitive to temperature. Considering the $4 \mathrm{~m} / \mathrm{s}$ entrainment viscosity case at $4 \%$ slip the traction coefficient with the best-fit Eyring model is 0.092 whereas the experimental measurement is 0.108 . The bulk temperature for that analysis is $68.2^{\circ} \mathrm{C}$, and repeat calculations with lower temperatures of $65^{\circ} \mathrm{C}$ and $60^{\circ} \mathrm{C}$ give calculated traction coefficients of 0.094 and 0.095 , respectively. The difference between best-fit model result and experiment is thus clearly greater than can be accounted for by bulk temperature changes of the components. 

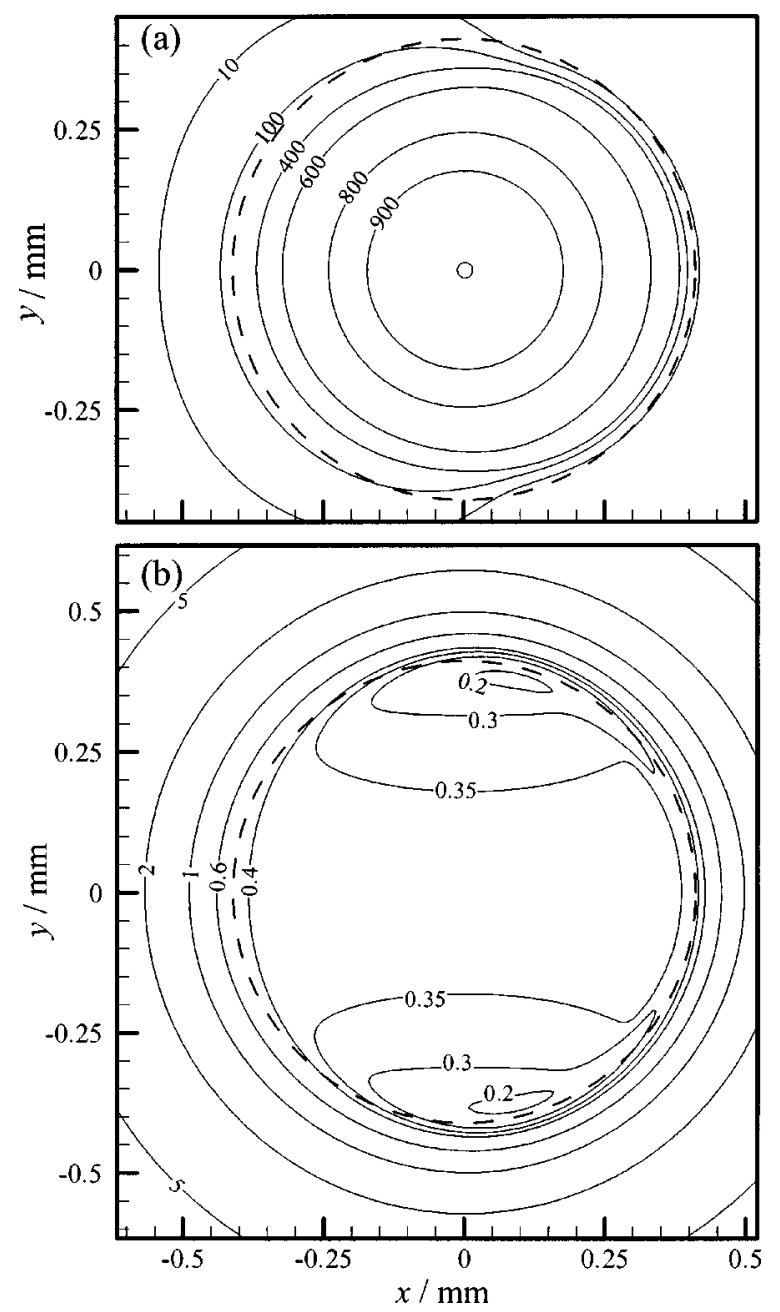

Fig. 11 Contours of (a) pressure/MPa, and (b) film thickness $/ \mu \mathrm{m}$ for the case $\hat{u}=11 \mathrm{~m} / \mathrm{s}, \theta_{\text {ref }}=90^{\circ} \mathrm{C}, \xi=0.01$. Broken circle indicates Hertzian contact area.

Since the fit at $90^{\circ} \mathrm{C}$ and $120^{\circ} \mathrm{C}$ is generally better, the fitting operation was repeated but without including the $60^{\circ} \mathrm{C}$ results and parameter values for this restricted fit are also given in Table 4. However, although the error measure was reduced this did not lead to any significant improvement in the traction curve fits as can be seen from the resulting best-fit curves shown in Fig. 10. A further factor considered was that of elastic shear behavior. This was included in trial models using an elastic shear modulus for the contact of $30 \tau_{L}$ as determined by Johnson [17], but no significant change resulted from this factor even when its value was reduced to $10 \tau_{L}$ to enhance its effect. Elastic shear is not therefore expected to be the source of the discrepancies between model and experiment.

During this investigation several-hundred EHL analyses were carried out and it became clear that the traction behavior depends crucially on the interaction between the shear stress developed in the model and the resulting temperature changes brought about through the thermal analysis. The feedback between the oil film temperature distribution and the viscosity was identified as the primary discriminating factor in the overall thermal EHL traction calculation, so that any uncertainty in viscosity/temperature behavior at high pressure is an important factor.

Figure 11 shows a typical EHL result obtained in the fitting exercise described above. Figure 11 shows contours of the pressure and film thickness distributions for the case with $\hat{u}=11 \mathrm{~m} / \mathrm{s}$ and $\theta_{\text {ref }}=90^{\circ} \mathrm{C}$. The model used for this analysis is the Eyring
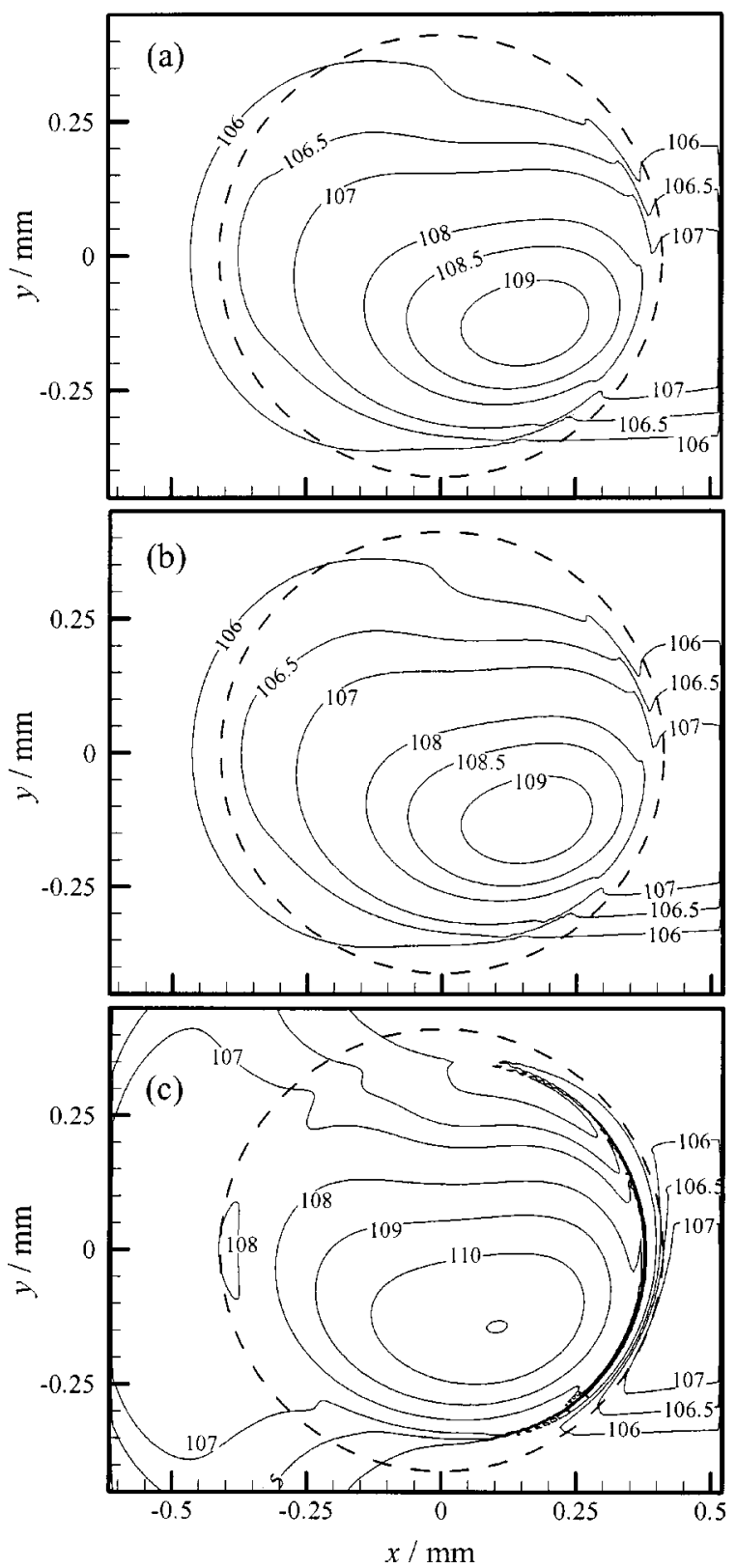

Fig. 12 Contours of temperature $/{ }^{\circ} \mathrm{C}$ for the case $\hat{u}=11 \mathrm{~m} / \mathrm{s}$, $\theta_{\text {ref }}=90^{\circ} \mathrm{C}, \xi=0.01$ with Eyring best fit model: (a) sphere surface temperature, (b) disk surface temperature, and (c) mid plane film temperature. Broken circle indicates Hertzian contact area.

form of Eq. (10) with a slide roll ratio $\xi=0.01$. The central film thickness value is $0.36 \mu \mathrm{m}$ and the minimum film thickness in the side lobes is $0.18 \mu \mathrm{m}$. The temperature distribution for the solution is shown in Fig. 12 which illustrates temperature contours for the upper (spherical) surface, the oil mid-plane (midway between the surfaces) and the lower (plane surface). The bulk temperature for this example is $105.6^{\circ} \mathrm{C}$, and the mid-plane oil film temperature can be seen to rise to $111^{\circ} \mathrm{C}$ within the contact. The maximum temperatures of the two components are seen to be $109^{\circ} \mathrm{C}$. For this case the asymmetry due to the spin motion is only apparent in the temperature distributions, and in each case the maximum temperature is located in the part of the contact with the highest sliding speed. The results are illustrated in the orientation that has the center of rotation of the disk at co-ordinate position $\left(0,-R_{t}\right)$. The case illustrated has a positive value of $\xi$ for which the sphere's 

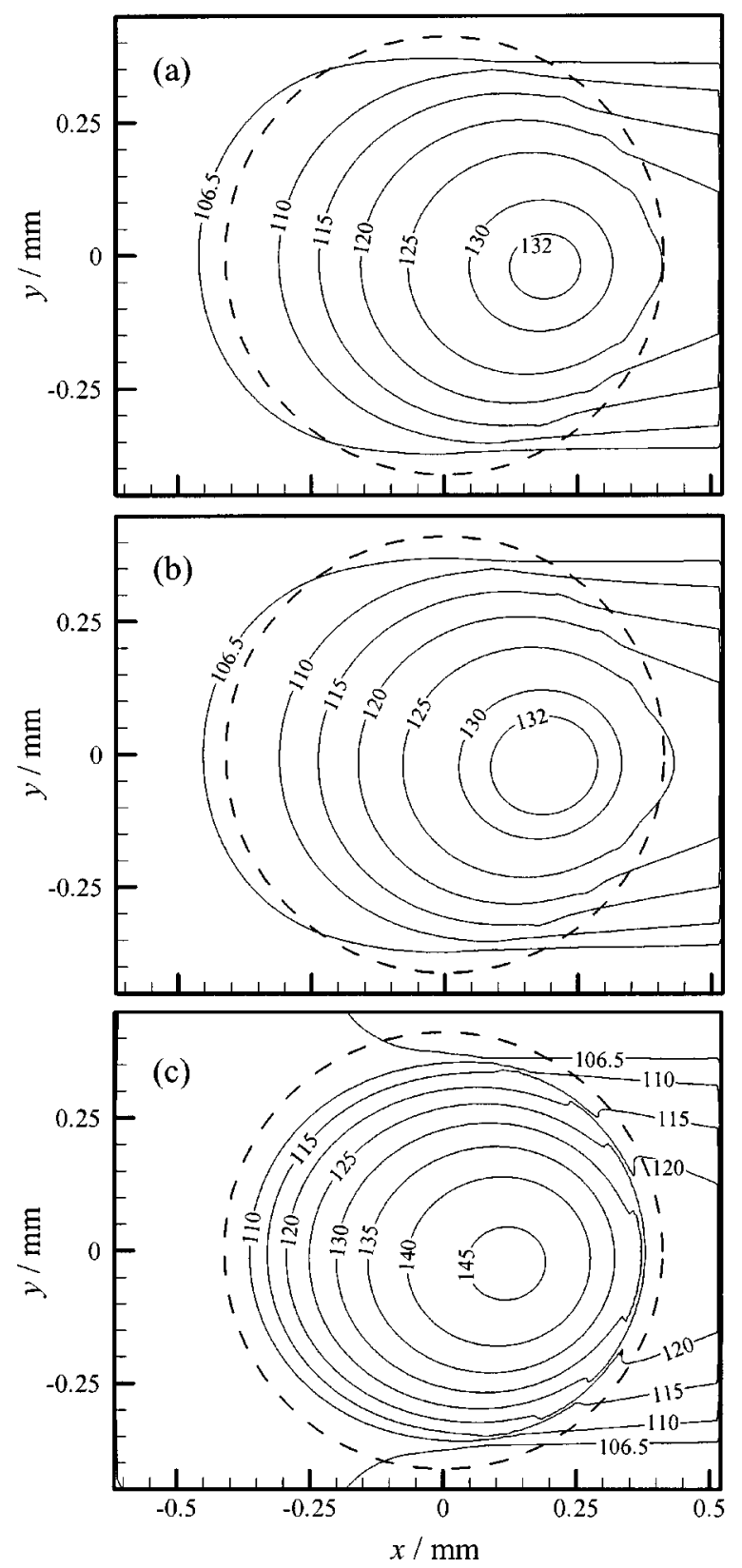

Fig. 13 Contours of temperature $/{ }^{\circ} \mathrm{C}$ for the case $\hat{u}=11 \mathrm{~m} / \mathrm{s}$, $\theta_{\text {ref }}=90^{\circ} \mathrm{C}, \xi=0.1$ with Eyring best fit model: (a) sphere surface temperature, $(b)$ disk surface temperature, and (c) mid-plane film temperature. Broken circle indicates Hertzian contact area.

velocity at the contact point $(0,0)$ is faster than the disk. Consequently, the variation in the velocity of the disk over the contact area means that the sliding speed increases as $y$ decreases.

As sliding is increased the changes in film thickness are small but the temperature rise changes considerably. Figure 13 illustrates the temperature contours for the same case but at a higher slide roll ratio of $\xi=0.1$. The differences in film thickness and pressure distributions between the cases with $\xi=0.01$ and 0.1 are too small to be visible by comparison of contour plots. The temperature differences are significant, however, as may be expected. The bulk temperature is now slightly higher at $106.2^{\circ} \mathrm{C}$ but the temperature rise seen in the oil film is much larger. The maximum temperature developed on the spherical surface is $132.7^{\circ} \mathrm{C}$ and the maximum value on the disk surface is $133.5^{\circ} \mathrm{C}$. For the case illustrated the kinematic configuration has the sphere moving faster
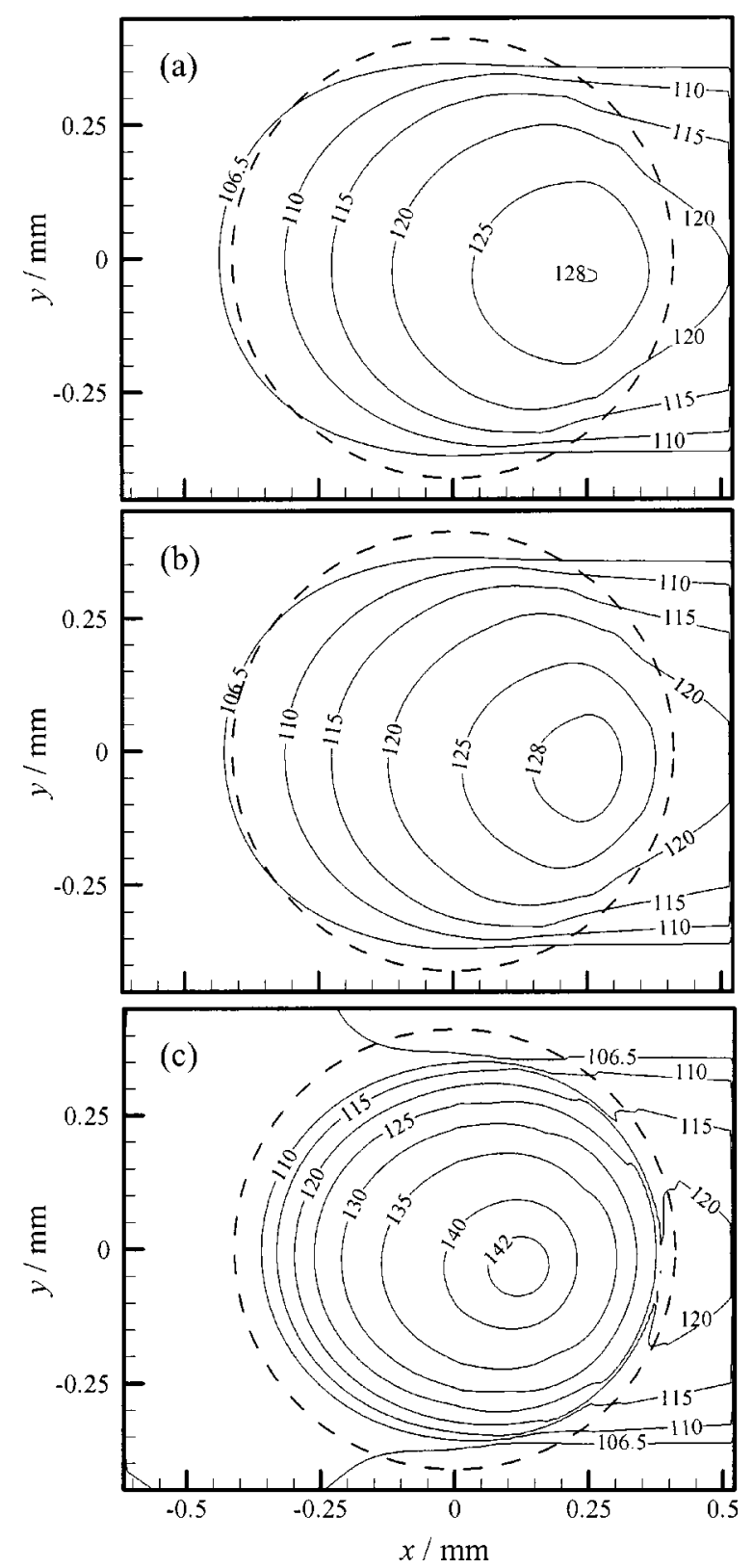

Fig. 14 Contours of temperature $/{ }^{\circ} \mathrm{C}$ for the case $\hat{u}=11 \mathrm{~m} / \mathrm{s}$, $\theta_{\text {ref }}=90^{\circ} \mathrm{C}, \xi=0.1$ with limiting shear stress best fit model: (a) sphere surface temperature, $(b)$ disk surface temperature, and (c) mid plane film temperature. Broken circle indicates Hertzian contact area.

at the contact point and the biggest temperature rise is associated with the slower moving surface. The mid plane film temperature is considerably higher at $146^{\circ} \mathrm{C}$. The increase in nominal sliding results in the spin differences within the contact becoming relatively less important, and as a result the temperature rise contours are more symmetric with respect to the $x$-axis at this higher slide roll ratio. The results illustrated in Figs. 11, 12, and 13 were obtained with the overall best fit model using Eq. (10). Figure 14 shows the corresponding result for $\xi=0.1$ obtained with the overall best fit model using Eq. (11). The maximum surface temperatures attained with this model are some $5^{\circ} \mathrm{C}$ lower at 128 and $129^{\circ} \mathrm{C}$, respectively. The maximum mid plane film temperature is $143^{\circ} \mathrm{C}$.

In the modeling work described above non-Newtonian constitutive model parameters have been determined by systematic fit- 
Table 5 Coefficients for Eq. (14) given in [18]

\begin{tabular}{ccccc}
\hline \hline$S_{1}$ & $S_{2}$ & $S_{3}$ & $T_{0}$ & $\eta_{0}$ \\
\hline $4.4 \times 10^{4}$ & $2.8 \times 10^{-8}$ & 0.162 & $38^{\circ} \mathrm{C}$ & $0.030 \mathrm{Pas}$ \\
& & & $99^{\circ} \mathrm{C}$ & $0.005 \mathrm{Pas}$ \\
\hline \hline
\end{tabular}

ting of full thermal EHL point contact analyses to experimental traction curves taken over a wide range of operating conditions and at the scale of traction drive contacts. Fang et al. [18] have carried out a different fitting exercise to experimental traction data. Their approach is different in that they have adopted an empirical viscosity formula of the form

$$
\eta(T, p)=S_{1} \eta_{0} \exp \left\{S_{2} p-S_{3}\left(T-T_{0}\right)\right\}
$$

in the fitting process, together with a fixed value for the Eyring stress $\tau_{0}$. The values of $S_{1}, S_{2}, S_{3}$, and $\tau_{0}$ were obtained for a given lubricant by fitting traction calculations to a range of traction experiments. The viscosity formula of Eq. (14) is stated by Fang et al. to be appropriate for the high pressure region corresponding to the Hertzian contact area where the traction force is generated. The values of the constants obtained in this exercise for Santotrac 50 are given in Table 5.

Attempts to use Eq. (14) to replace Eq. (9) in the traction modeling analysis were unsuccessful. This is because the low pressure viscosity behavior given by equation (14) is significantly different to the measured viscosity behavior because of the large value of factor $S_{1}$. The low pressure viscosity behavior determines the film thickness and therefore the shear rate experienced by the fluid in the contact area. Using Eq. (14) would therefore cause the numerical model to develop oil films that are wholly unrealistic so that any traction modelling, in the sense of that carried out in the current study, would be futile.

\section{Conclusions}

Using the Yasutomi et al. [15] viscosity/pressure/temperature relation for Santotrac 50 traction fluid and a range of nonNewtonian models has enabled theoretical traction curves to be determined for a wide range of conditions corresponding to traction experiments. Constants that determine the temperature behavior of the relevant non-Newtonian shear stress parameter(s) have been evaluated using a best-fit approach to the low slip $(<4 \%)$ region of the traction curves. The best-fit defined in this way is obtained with an Eyring type model. This gives a reasonably accurate working model up to the slip level of 5\% which characterizes safe traction drive behavior. Its deficiencies in this range are effectively a conservative evaluation of the available traction at the lowest entrainment velocity considered.

A linear temperature variation of the non-Newtonian parameters has been assumed in the investigation, and work to extend consideration to a nonlinear temperature dependence such as that shown in Fig. 7 is in progress.

\section{Acknowledgments}

The authors gratefully acknowledge the support of EPSRC (award GR/N36301/01) and the support for the LAMTRAK project (ND21/49) under the FORESIGHT VEHICLE SCHEME.

\section{Nomenclature}

$A=$ area subject to lubricant pressure, $\mathrm{m}^{2}$

$A, B, C, D=$ constants used in formulas for $\tau_{0}$ and $\tau_{L}$

$A_{k}, B_{k}=$ pressure and film thickness coefficients in discretised Reynolds equation

$C_{k}, D_{k}=$ pressure and film thickness coefficients in discretised deflection equation

$c=$ specific heat, $\mathrm{J} / \mathrm{kgK}$

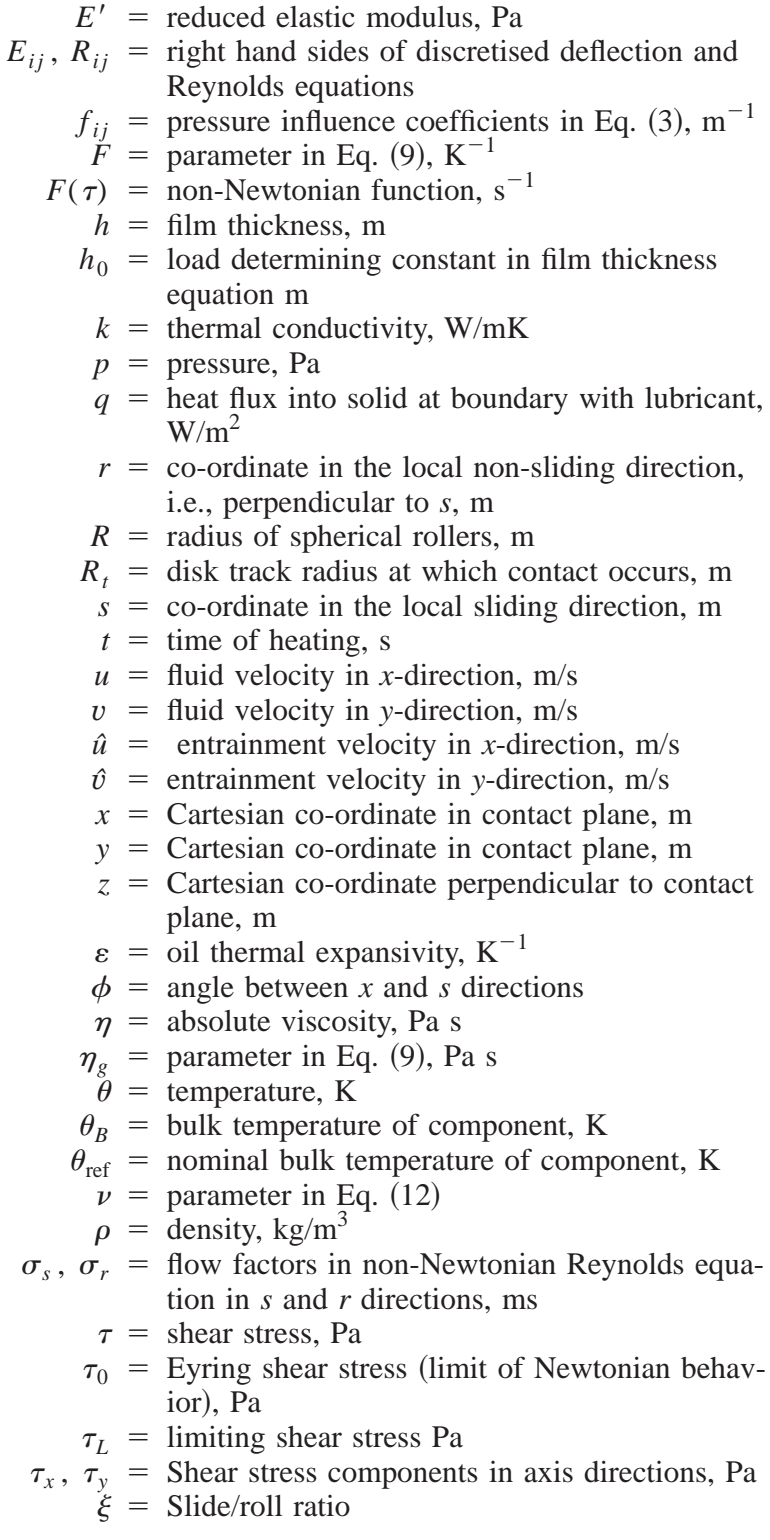

\section{References}

[1] Heumann, H., Briffet, G., Burke, M., Field, M., Fuller, J., Lee, A. P., and Newall, J. P., 2002, "System Efficiency Optimization of the Torotrak Infinitely Variable Transmission (IVT),"CVT 2002 Congress, VDI, Munich, October 2002.

[2] Dyson, A., 1970, "Frictional Traction and Lubricant Rheology in Elastohydrodynamic lubrication," Philos. Trans. R. Soc. London, A266, pp. 1-33.

[3] Hirst, W., and Moore, A. J., 1974, "Non-Newtonian Behavior in Elastohydrodynamic Lubrication,” Proc. R. Soc. London, Ser. A, 337, pp. 101-121.

[4] Johnson, K. L., and Tevaarwerk, J. L., 1977, "The Shear Behavior of Elastohydrodynamic Oil Films," Proc. R. Soc. London, Ser. A, 356, pp. 215-236.

[5] Conry, T. F., Wang, S., and Cusano, C., 1987, "A Reynolds-Eyring Equation for Elastohydro-Dynamic Lubrication in Line Contacts," ASME J. Tribol., 109, pp. 648-654.

[6] Kim, K. H., and Sadeghi, F., 1991, "Non-Newtonian Elastohydro-Dynamic Lubrication of Point Contact,” ASME J. Tribol., 113, pp. 703-711.

[7] Sharif, K. J., Kong, S., Evans, H. P., and Snidle, R. W., 2001, "Contact and Elastohydrodynamic Analysis of Worm Gears: Part 1 Theoretical Formulation," Proc. Inst. Mech. Eng., Part C: J. Mech. Eng. Sci., 215, pp. 817-830.

[8] Bair, S., and Winer, W. O., 1979, "A Rheological Model for Elastohydrodynamic Contacts Based on Primary Laboratory Data," ASME J. Lubr. Technol., 101, pp. 258-265.

[9] Bair, S., and Winer, W. O., 2000, "The Pressure-Viscosity Coefficient at Hertz Pressure and Its Relation to Concentrated Contact Traction," Proc. 26th LeedsLyon Symp. on Tribology, Elsevier, Amsterdam pp. 433-443.

[10] Newall, J. P., Nicolson, D. M., Lee, A. P., and Evans, S. P., 2002, “Development and Assessment of Traction Fluids for Use in Toroidal (IVT) Transmissions," SAE 2002 World Congress, Transmissions and Drive-lines Symposium, March 2002. 
[11] Plint, M. A., 1967, “Traction in Elastohydrodynamic Contacts," Proc. I. Mech. E., 182, n14, pp. 300-306.

[12] Holmes, M. J. A., Evans, H. P., Hughes, T. G., and Snidle, R. W., 2003, "Transient Elastohydrodynamic Point Contact Analysis Using a New Coupled Differential Deflection Method: Part 1 Theory and Validation," Proc. Inst. Mech. Eng., Part J: J. Eng. Tribol. 217, pp. 289-303.

[13] Evans, H. P., and Hughes, T. G., 2000, "Evaluation of Deflection in SemiInfinite Bodies by a Differential Method," Proc. Inst. Mech. Eng., Part C: J. Mech. Eng. Sci., 214, pp. 563-584.

[14] Larsson, R., Larsson, P. O., Eriksson, E., Sjöberg, M., and Höglund, E., 2001, "Lubricant Properties for Input to Hydrodynamic and ElastohydroDynamic Lubrication Analyses,” Proc. Inst. Mech. Eng., Part J: J. Eng. Tribol., 201, pp. 17-28.
[15] Yasutomi, S., Bair, S., and Winer, W., 1984, “An Application of a Free Volume Model to Lubricant Rheology,” ASME J. Tribol., 106, pp. 291-303.

[16] Evans, C. R., and Johnson, K. L., 1986, "The Rheological Properties of Elastohydrodynamic Lubricants," Proc. Inst. Mech. Eng., Part C: Mech. Eng. Sci., 200, pp. 303-312.

[17] Johnson, K. L., 1993, "Non-Newtonian Effects in Elastohydro-Dynamic Lubrication," Thin Films in Tribology, Proc. 19th Leeds-Lyon Symposium, Elsevier, Amsterdam, pp. 25-26.

[18] Fang, N., Chang, L., Johnson, G. J., Webster, M. N., and Jackson, A., 2001, "An Experimental/Theoretical Approach to Modelling the Viscous Behavior of Liquid Lubricants in Elastohydrodynamic Lubrication Contacts," Proc. Inst. Mech. Eng., Part J: J. Eng. Tribol., 215, pp. 311-319. 